1999 Particle Accelerator Conference, New York, NY, March 29-April 2, 1999

\title{
BNL-BUILT LHC MAGNET ERROR IMPACT ANALYSIS AND COMPENSATION*
}

\author{
V. Ptitsin ${ }^{*}$, S. Tepikian, J. Wei, BNL, Upton, NY
}

\section{Abstract}

Superconducting magnets built at the Brookhaven National Laboratory will be installed in both the Insertion Region IP2 and IP8, and the RF Region of the Large Hadron Collider (LHC). In particular, field quality of these IR dipoles will become important during LHC heavy-ion operation when the $\beta^{*}$ at IP2 is reduced to 0.5 meters. This paper studies the impact of the magnetic errors in BNL-built magnets on LHC performance at injection and collision, both for proton and heavy-ion operation. Methods and schemes for error compensation are considered including optimization of magnet orientation and compensation using local IR correctors.

\section{INTRODUCTION}

An important part of the USA contribution to the Large Hadron Collider (LHC) project is the construction of superconducting magnets for the interaction and $\mathrm{RF}$ regions of the future collider. The half of the total number of IR triplet quadrupole magnets will be produced by FNAL which is also responsible for the assembling another half of IR quads produced by KEK into cryostat. The BNL constructs superconducting dipole magnets for $\mathrm{LHC}$ experimental and RF insertions.

In the previous paper[1] we analysed the impact of magnet field errors in the IR quadrupoles and dipoles on LHC collision performance for proton collisions at top energy. In this article the accent is made on the evaluation of the impact coming from the errors of BNL-made dipoles both at collision and injection and on the case of heavy ion collision lattice with additional low-beta interaction point at IP2 in comparison with the proton collision lattice. The basic parameters of proton and ion lattices used at the analysis are listed in Table 1.

Table 1: Lattice parameters.

\begin{tabular}{llll}
\hline Quantity & p inj. & p coll. & ion coll. \\
\hline $\mathrm{E}[\mathrm{GeV}]$ & 450 & 7000 & 7000 per charge \\
$\nu_{x} / \nu_{y}$ & $63.28 / 59.31$ & $63.31 / 59.32$ & $63.31 / 59.32$ \\
$\xi_{x} / \xi_{y}$ & $2 / 2$ & $2 / 2$ & $2 / 2$ \\
$\epsilon_{N}[\mathrm{~m} \cdot \mathrm{r}]$ & $3.75 \times 10^{-6}$ & $3.75 \times 10^{-6}$ & $1.5 \times 10^{-6}$ \\
$\sigma_{p}$ & $4.7 \times 10^{-4}$ & $1.1 \times 10^{-4}$ & $1.14 \times 10^{-4}$ \\
\hline
\end{tabular}

Two beam dynamic characteristic quantities are used to evaluate the error impact: the dynamic aperture (DA) and the tune footprint. The target values for the LHC are more than $12 \sigma$ of average DA, more than $10 \sigma$ minimum DA and less than $10^{-3}$ tune spread.

\footnotetext{
*Email: vadimp@bnl.gov

*Work performed under the auspices of the
}

\section{TRACKING SETUP}

Fortran version of TEAPOT code was used for tracking studies. We restricted our investigation to 1000 turn tracking. Previous study indicates that $10^{5}$ turn tracking further reduces DA by $0.5-1 . \sigma$.

Ten seeds of magnet errors were created based on the error tables for all studied magnets (the errors of warm D1 dipoles at IR1 and IR5 also were included). We excluded from the consideration only sqew quadrupole component of the errors assuming that the coupling is completely compensated.

The working point at collision $\left(\nu_{x}=63.31, \nu_{y}=59.32\right)$ and injection $\left(\nu_{x}=63.28, \nu_{y}=59.31\right)$ are both close enough to the third integer resonance condition. Thus to avoid the decrease in dynamic aperture the machine has to be retuned to the nominal working point after the magnet errors are introduced to the lattice. Also the arc sextupoles are used to correct the chromaticity to the nominal values of 2.0 in both $X$ and $Y$ planes.

The tracking is 6 dimensional with the RF system operating at nominal values of RF voltage. The particles to track are taken with the initial $2.5 \sigma$ energy deviation.

\section{DIPOLE ERROR ANALYSIS}

BNL superconducting dipoles include interaction region beam separation magnets D1 for IR2 and IR8, interaction region magnets D2 for all $4 \mathrm{IRs}$, and RF insertion magnets D3A, D3B, D4A, D4B (Fig.1). The magnet design is based on the RHIC arc dipole. However, due to the specific LHC requirements the magnets D2,D4A, D4B utilize 2-in-1 design with two magnet apertures in one yoke. Examples of 1-in-1 and 2-in-1 magnet designs fro BNL-built dipoles are shown in Figure 2.

The expected dipole error sets for D1 and D3 magnets at injection and collision used at the analysis are shown in Tables 2, 3 where $b_{2}, a_{2}$ present quadrupole components. The twin aperture magnets (D2,D4) have slightly different the error set with $b_{3}=-5.7$ at injection and $b_{3}=-0.48$ at collision.

The big value of $b_{3}$ at injection is caused by the effect of persistent current. In comparison with RHIC case this effect is relatively large. The magnet design was optimized initially for RHIC but the current of RHIC magnet at injection is about $600 \mathrm{~A}$ while the magnets for LHC would use the current as low as $300 \mathrm{~A}$ which leads to increasing persistent sextupole component. The analysis showed the persistent $b_{3}$ at the arc LHC dipoles is of the same value as shown in Table 4. The contribution of the the RF region dipoles $(\mathrm{D} 3, \mathrm{D} 4)$ to the chromaticity variation is small in comparison with the total contribution from the large 
Table 2: Expected D1 \& D3 errors at collision. $R_{0}=17$

\begin{tabular}{llllllr} 
mm & \multicolumn{7}{c}{ Normal } \\
\hline$n$ & $\left\langle b_{n}\right\rangle$ & $d\left(b_{n}\right)$ & $\sigma\left(b_{n}\right)$ & $\left\langle a_{n}\right\rangle$ & $d\left(a_{n}\right)$ & $\sigma\left(a_{n}\right)$ \\
\hline Body & [unit] & & & & & \\
2 & 0.07 & 0.54 & 0.19 & 0.43 & 2.4 & 1.1 \\
3 & -1.5 & 1.6 & 0.84 & -0.12 & 0.27 & 0.10 \\
4 & 0.00 & 0.08 & 0.03 & 0.01 & 0.34 & 0.13 \\
5 & 0.11 & 0.17 & 0.09 & -0.01 & 0.04 & 0.01 \\
7 & 0.11 & 0.02 & 0.01 & -0.00 & 0.01 & 0.00 \\
9 & 0.00 & 0.01 & 0.00 & -0.00 & 0.00 & 0.00 \\
LE & [unit $\mathrm{m}$ ] & \multicolumn{7}{c}{ (Length=0.73 m) } & \\
2 & -0.3 & 1.5 & 0.7 & -1.0 & 2.9 & 1.2 \\
3 & 10.3 & 1.4 & 0.5 & -4.6 & 0.5 & 0.2 \\
5 & -0.1 & 0.2 & 0.1 & 0.5 & 0.1 & 0.0 \\
RE & [unit·m] & \multicolumn{5}{c}{ (Length=0.73 m) } \\
2 & 0.2 & 1.2 & 0.5 & 0.6 & 3.1 & 1.3 \\
3 & 2.8 & 1.2 & 0.5 & 0.1 & 0.5 & 0.2 \\
\hline
\end{tabular}

amount of arc dipole magnets and does not require special corrections. Furthemore, no noticable impact was found from the saturation of $b_{3}$ component at collision.

Compensation measures have been taken to diminish the effect of the errors ofinteraction region dipole D1 on beam dynamics. They include:

1. Magnet Orientation Optimization: D1 lead end is oriented towards the interaction points where a $b_{3}$ corrector is located.

2. Body-End Compensation for the systematic $b_{3}$ :

$b_{3}($ Body $)=-0.095 B_{3}(\mathrm{LE})-0.116 B_{3}(\mathrm{RE})=-1.3[\mathrm{u}]$

Tracking studies were performed for the proton LHC lattice both at injection and collision with the whole set of errors from dipole error tables. The studies indicated no CMS

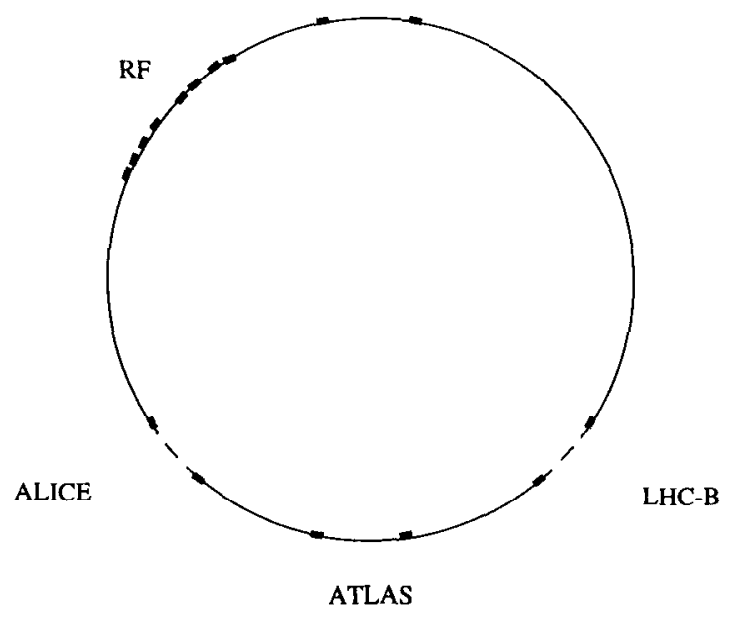

D2 D3A, D3B, D4A, D4B

Figure 1: BNL-made dipoles at LHC ring
Table 3: Expected D1 \& D3 errors at injection. $R_{0}=17$ $\underline{m m}$

\begin{tabular}{|c|c|c|c|c|c|c|}
\hline \multirow[t]{2}{*}{$n$} & \multicolumn{3}{|c|}{ Normal } & \multicolumn{3}{|c|}{ Skew } \\
\hline & $\left\langle b_{n}\right\rangle$ & $d\left(b_{n}\right)$ & $\sigma\left(b_{n}\right)$ & $\left\langle a_{n}\right\rangle$ & $d\left(a_{n}\right)$ & $\sigma\left(a_{n}\right)$ \\
\hline Body & [unit] & & & & & \\
\hline 2 & 0.08 & 0.51 & 0.19 & 0.14 & 2.8 & 1.1 \\
\hline 3 & -6.3 & 2.5 & 0.92 & -0.03 & 0.24 & 0.09 \\
\hline 4 & -0.02 & 0.07 & 0.03 & 0.04 & 0.37 & 0.13 \\
\hline 5 & 0.14 & 0.18 & 0.09 & -0.01 & 0.04 & 0.01 \\
\hline 7 & -0.04 & 0.02 & 0.01 & 0.0 & 0.01 & 0.0 \\
\hline 9 & 0.01 & 0.01 & 0.0 & 0.0 & 0.0 & 0.0 \\
\hline LE & [unit $\cdot \mathrm{m}]$ & & (Lengt & $=0.73$ & & \\
\hline 2 & -0.2 & 1.5 & 0.7 & -1.6 & 2.9 & 1.1 \\
\hline 3 & 8.7 & 1.3 & 0.5 & -4.6 & 0.5 & 0.2 \\
\hline 5 & -0.1 & 0.2 & 0.1 & 0.5 & 0.1 & 0.0 \\
\hline RE & [unit $\cdot \mathbf{m}]$ & & (Lengt & $=0.73$ & & \\
\hline 2 & 0.2 & 1.3 & 0.5 & -0.2 & 3. & 1.1 \\
\hline 3 & 1.8 & 1.1 & 0.5 & 0.1 & 0.5 & 0.2 \\
\hline
\end{tabular}

Table 4: Persistent current contribution from D3.D4 dipoles versus arc dipoles

\begin{tabular}{lcc}
\hline Quantity & Arc dipoles & D3,D4 \\
\hline Persistent $b_{3}[\mathrm{u}]$ & -9 & -6 \\
Dispersion $[\mathrm{m}]$ & 1.5 & 0.1 \\
Chromaticity & 500 & 0.03 \\
\hline
\end{tabular}

noticeable impact of the dipole errors on the beam dynamics. If only BNL dipole errors are taken into the account the resulting dynamic aperture is beyond the physical aperture. Figure 3 shows no difference in $11 \sigma$ footprint at the injection energy for the cases with and without the BNL dipole errors.

\section{HEAVY-ION OPERATION}

Heavy-ion collision lattice uses low- $\beta^{*}$ IP2 in addition to low- $\beta^{*}$ collisions at IP1 and IP5. This produces large values of $\beta$ functions in corresponding IR triplet quadrupoles and D1 dipoles. Furthemore, all interaction regions utilize orbit separation and crossing angle schemes. Such schemes lead to large orbit excursion inside IR quads and dipoles thus shifting the beam to the field regions with larger nonlinearity. The interaction region configuration for ion lattice used at the tracking studies is shown in Table 5 . The worst case is considered with the crossing angle of $\pm 150 \mu \mathrm{rad}$ at each low $\beta$ interaction points.

The tracking used the two possible schemes of IR
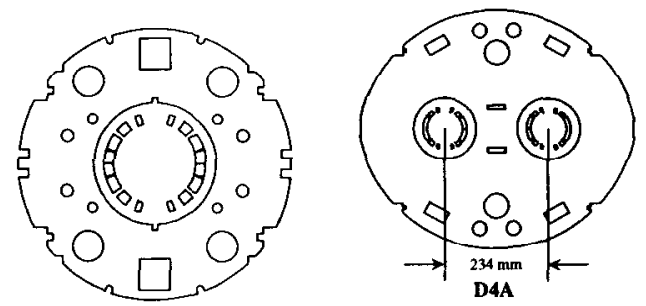

Figure 2: Magnet body cross section of BNL-built D1 and D4A. 

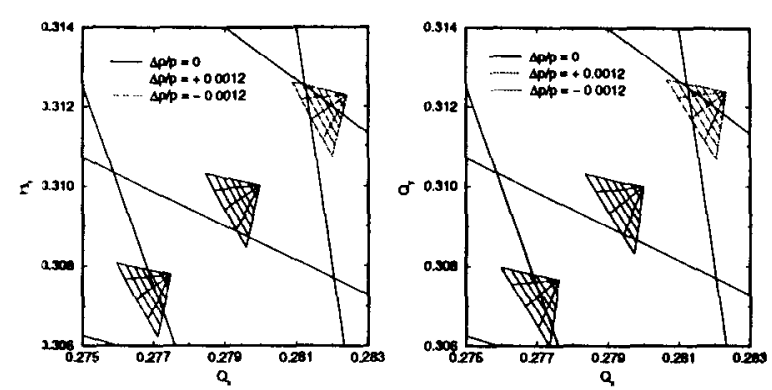

Fiqure 3: $11 \sigma$ tune footprints with and without the errors in D1,D2,D3,D4 magnets.

Table 5: Interaction region configuration parameters

\begin{tabular}{|l|c|c|c|c|}
\hline & IP1 & IP2 & IP5 & IP8 \\
\hline sep. $[\mathrm{mm}]$ & 0 & 0 & 0 & $1.5 \mathrm{~h}$ \\
\hline agle $[\mu \mathrm{rad}]$ & $\pm 150 \mathrm{v}$ & $\pm 150 \mathrm{v}$ & $\pm 150 \mathrm{~h}$ & $\pm 100 \mathrm{v}$ \\
\hline$F_{x}^{*}, \beta_{y}^{*}[\mathrm{~m}]$ & $0.5,0.5$ & $0.5,0.5$ & $0.5,0.5$ & 33,33 \\
\hline
\end{tabular}

quatrupole arrangement. In the unmixed scheme KEKbuilt magnets are installed at IR1, IR2 and FNAL-built mognets at IR5, IR8. In the mixed scheme the each interaction region contains both KEK-built $(Q 1, Q 3)$ and FNALbuilt (Q2A,Q2B) quadrupoles. The most tracking has been dowe for the mixed scheme. Table 6 presents a summary of the tracking results.

Table 6: Effects of MQX and dipole errors in terms of $10^{3}$ turm 6D DA.

\begin{tabular}{lll}
\hline Case & DA $\left(\sigma_{x y}\right)$ & Min. DA \\
\hline Unmixed case: & & \\
Full error & $8.3 \pm 1.8$ & $5 \sigma_{x y}$ \\
Errors at IR2 only & $9.7 \pm 2.4$ & $6 \sigma_{x y}$ \\
Quad error at IR2 only & $11.8 \pm 3.7$ & $6 \sigma_{x y}$ \\
\hline Mixed case: & & \\
Full error & $8.5 \pm 1.5$ & $5 \sigma_{x y}$ \\
Full error, no cross.angle & $13.1 \pm 2.1$ & $9 \sigma_{x y}$ \\
Quad error only & $8.9 \pm 1.6$ & $6 \sigma_{x y}$ \\
Errors at IR2 only & $10.2 \pm 2.3$ & $6 \sigma_{x y}$ \\
Quad error at IR2 only & $11.7 \pm 3.5$ & $6 \sigma_{x y}$ \\
Systematic only & $9.5 \pm 0.8$ & $8 \sigma_{x y}$ \\
Random only & $12.4 \pm 2.2$ & $8 \sigma_{x y}$ \\
Without $n=3,4$ errors & $9.1 \pm 1.8$ & $6 \sigma_{x y}$ \\
Without $n=5,6$ errors & $11.4 \pm 1.4$ & $7 \sigma_{x y}$ \\
Without $n=7,8$ errors & $8.1 \pm 2.5$ & $5 \sigma_{x y}$ \\
Without $n=9,10$ errors & $9.0 \pm 1.7$ & $6 \sigma_{x y}$ \\
IR dipole error only & $>$ physic.apert. & \\
\hline
\end{tabular}

The beam dynamic characteristics are mainly determined by the errors in IR quadrupoles. The dipoles, especially cold D1 at IR2, enhance the impact by further reducing the DA by up to $2 \sigma$. The mixed quad arrangement scheme provides slightly better results for DA as well as for the $6 \sigma$ footrpint size:

\begin{tabular}{lcc}
\hline scheme & footprint $\left[10^{-3}\right]$ & max.footprint $\left[10^{-3}\right]$ \\
\hline mixed & $2.7 \pm 1.5$ & 4.9 \\
separated & $3.6 \pm 1.9$ & 6.6 \\
\hline
\end{tabular}

The important case is when the errors were installed only at IR2 quads and dipoles. The resulting average DA of
$10.2 \sigma$ and minimum DA of $6 \sigma$ are below the acceptable values. It indicales that the non-linear correctors would also be useful at IR2 in order to achieve the target value of DA at the ion operation. The rms values of the results for DA with the errors at IR2 only are quite large. It is explained by the big difference between horizontal and vertical dynamic aperture in this case. Vertical DA is about 4-5 $\sigma$ smaller.

In order to understand how effectively the local IR correction schemes compensate for the IR magnet errors the tracking has been done with the non-linear correctors on. The present correction strategy includes 3 correctors per each triplet at IR1 and IR5. It is still under discussions and studies what the best choice of the layers of correction elements to be put into these correctors. The following corrector configuration was considered:

\begin{tabular}{lc}
\hline corrector place & harmonic layers \\
\hline between Q2A and Q2B & $b_{4}, b_{5}$ \\
between Q2B and Q3 & $a_{3}, a_{4}, a_{6}$ \\
between Q3 and D1 & $b_{3}, b_{6},\left(b_{10}\right)$ \\
\hline
\end{tabular}

In order to compensate for IR2 magnet errors the same corrector scheme was put also into IR2. The results obtained for the cases with and without correctors at IR2 confirmed the conclusion that the presence of the correctors at IR2 considerably improves the nonlinear dynamics characteristics:

\begin{tabular}{lcc}
\hline Case & $\overline{D A}\left(\sigma_{x y}\right)$ & Min. DA \\
\hline Correctors only at IR1,IR5 & $10.5 \pm 3.0$ & $6 \sigma_{x y}$ \\
With IR2 correctors & $17.0 \pm 1.7$ & $13 \sigma_{x y}$ \\
\hline
\end{tabular}

\section{CONCLUSION}

Field quality of BNL dipoles is adequate for nominal proton operation both for injection and collision lattices.

In the case of the ion lattice the contribution from IR2 magnets are considerable. The use of the non-linear correctors at IR2 is preferable if the crossing angle as large as $\pm 150 \mu \mathrm{rad}$ to be used.

\section{AKNOWLEDGEMENTS}

We thank J.-P. Koutchouk, O. Brüning and R. Ostojic for lattice assistance and discussions, and many others, including A. Jain, M. Harrison, S. Peggs, S. Plate, J. Strait, R. Talman and $E$. Willen.

\section{REFERENCES}

[1] J. Wie et al., "US-LHC IR magnet error analysis and compensation”, EPAC 1998 Proceedings, (1998) p.380.

[2] J. Wei et al., "Insertion region local correction for the Large Hadron Collider", these proceedings. 